\title{
ISW for the Treatment of Adult Skeletal Class III with Facial Asymmetry: A Case Report Crowding
}

\author{
Chun Shuo Huang, Jian Hong YU* and Yuan Hou CHEN \\ ${ }^{1}$ Department of Orthodontics, China Medical University and Medical Center, Taiwan
}

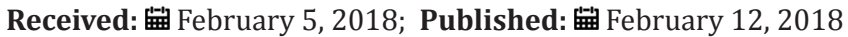

*Corresponding author: Jian Hong YU, Department of Orthodontics, China Medical University and Medical Center, Taiwan

\begin{abstract}
The objective of this case report was to discuss LH wire (Improved Super-elastic Ti-Ni alloy Wire) for the treatment of adult skeletal class III with facial asymmetry. An adult female aged 27 years 10 months who came to our clinic with a chief complaint of uneven occlusal plane and an obvious asymmetrical face. Clinical examination by functional wax bite reveals a possibility of functional interference around the right upper canine. P-A view check finds slight discrepancy between the right and the left ramus heights. Orthognathic surgery was suggested to the patient but was refused. After midline assumption, the patient was pleased with the outcome and thus orthodontic treatment was performed. After one year of active treatment, a stable occlusion was achieved, yet the asymmetrical appearance was not much improved immediately after the treatment. After a retention period of 6 months by a functional appliance to maintain the intermaxillary relationship and to adjust the musculature tension, a new balanced facial appearance was observed.
\end{abstract}

\section{Introduction}

The treatment of adult skeletal class III with facial asymmetry is usually a challenge to the orthodontist. It's important to differentially diagnose a facial asymmetry case by dental, functional, or skeletal problems. Clinical examination by functional wax bite reveals a possibility of functional interference around the right upper canine. P-A view check finds slight discrepancy between the right and the left ramus heights. Orthognathic surgery was suggested to the patient but was refused. After midline assumption, the patient was pleased with the outcome and thus orthodontic treatment was performed.

\section{History and Diagnosis}

An adult female who was aged 27 years 10 months who came to our clinic with a chief complaint of uneven occlusal plane and an obvious asymmetrical face. Her lateral profile was concave and the frontal view showed facial asymmetry (Figure 1). Her occlusion showed Class III molar and Class I canine relationships over right side and Class I canine relationship over left side. Her oral hygiene and gingival conditions were poor. \#36 missing, \#37 large decay, \#46 proximal caries were noted (Figures $2 \& 3$ ). Clinical examination by functional wax bite reveals a possibility of functional interference around the right upper canine. The cephalometric analysis showed a skeletal class III jaw relationships (SNA:81.4o, SNB:87.3o, ANB:5.9o) and dental compensation (U-1 to FH plane : 131.80 , L-1 to mandibular plane: 84.5o). P-A (postero-anterior) cephalometric radiograph showed slight discrepancy between the right and the left ramus heights (Figures 4-6) (Table 1).

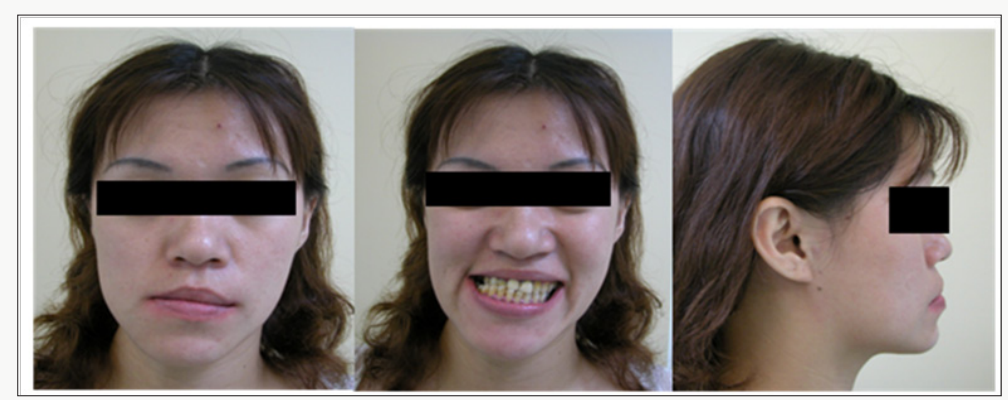

Figure 1: Facial photos before active treatment. 


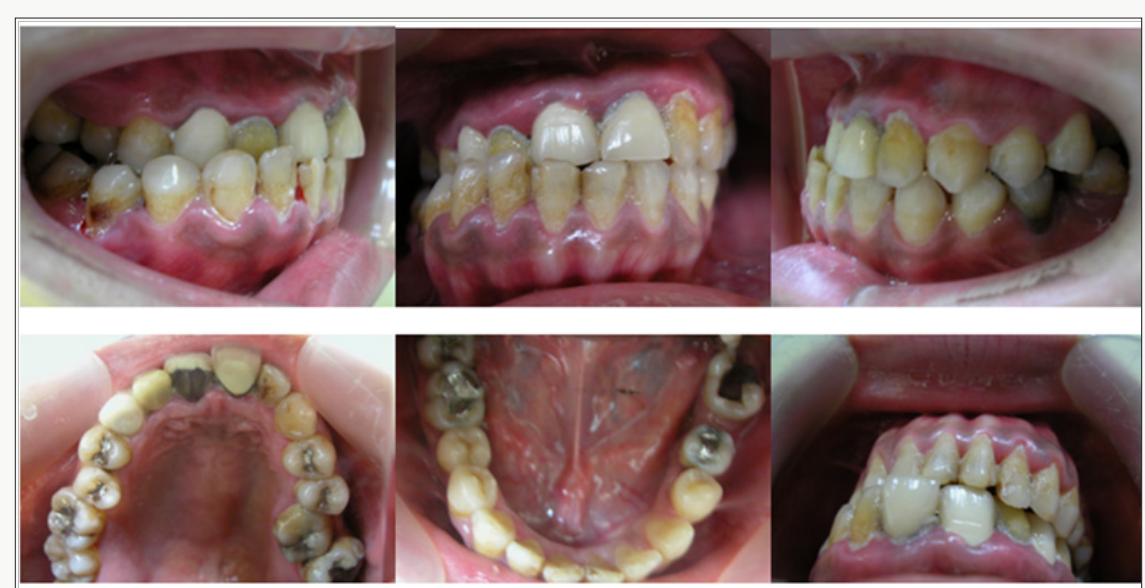

Figure 2: Intraoral photos before active treatment.

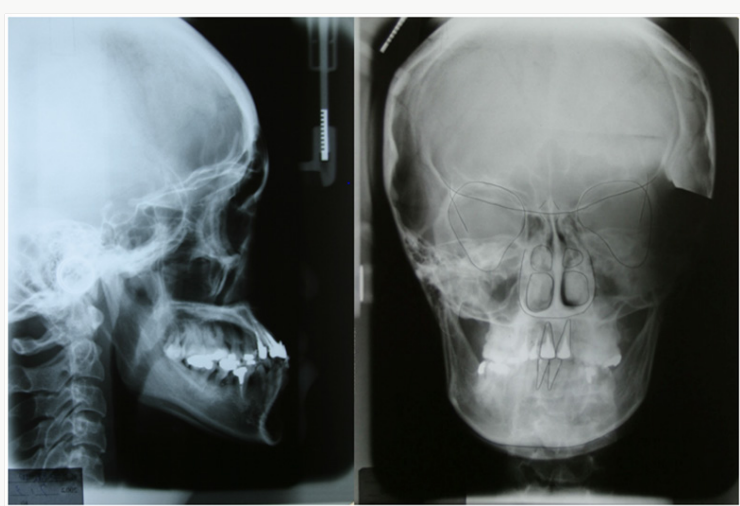

Figure 3: Pretreatment lateral and PA (postero-anterior) cephalometric radiographs.

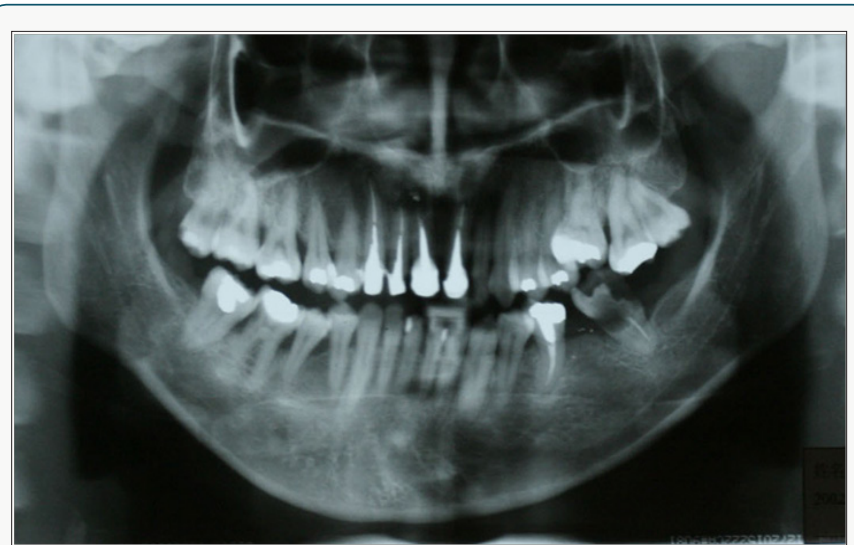

Figure 4: Pretreatment panoramic radiographs.

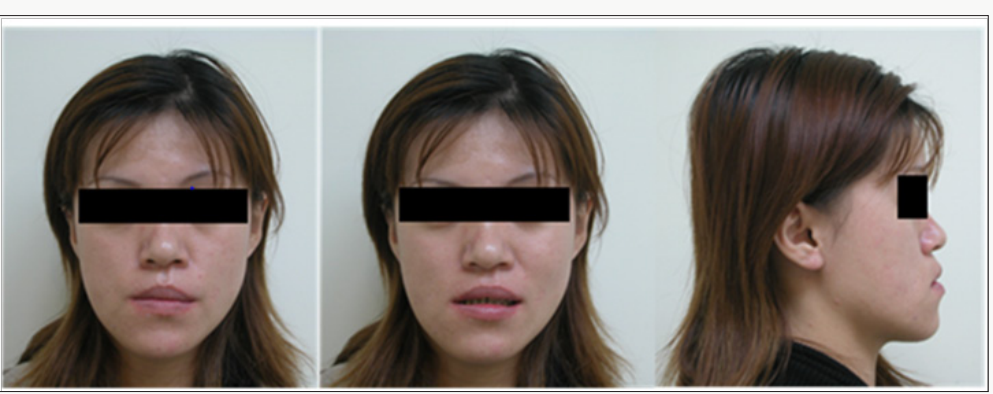

Figure 5: Facial photos after active treatment.

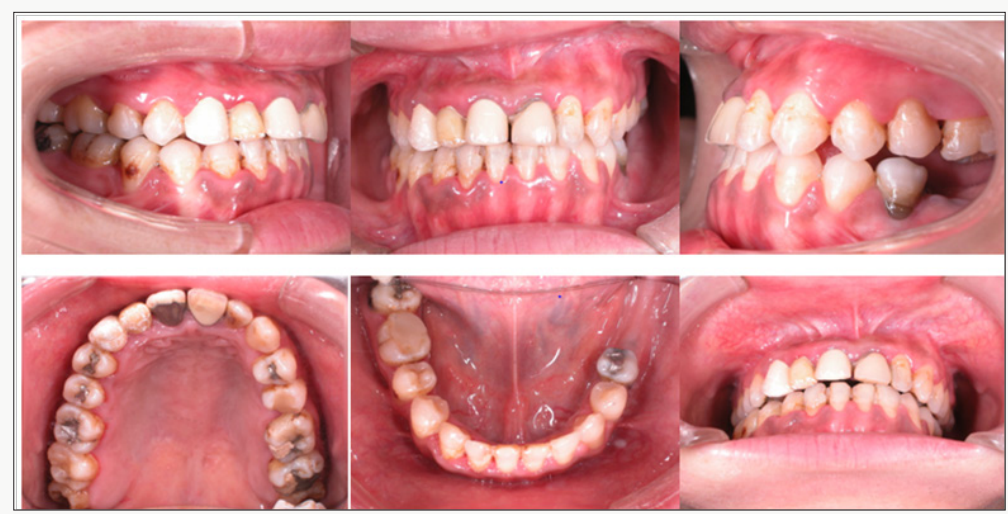

Figure 6: Intraoral photos after active treatment. 
Table 1: Polygon before active treatment.

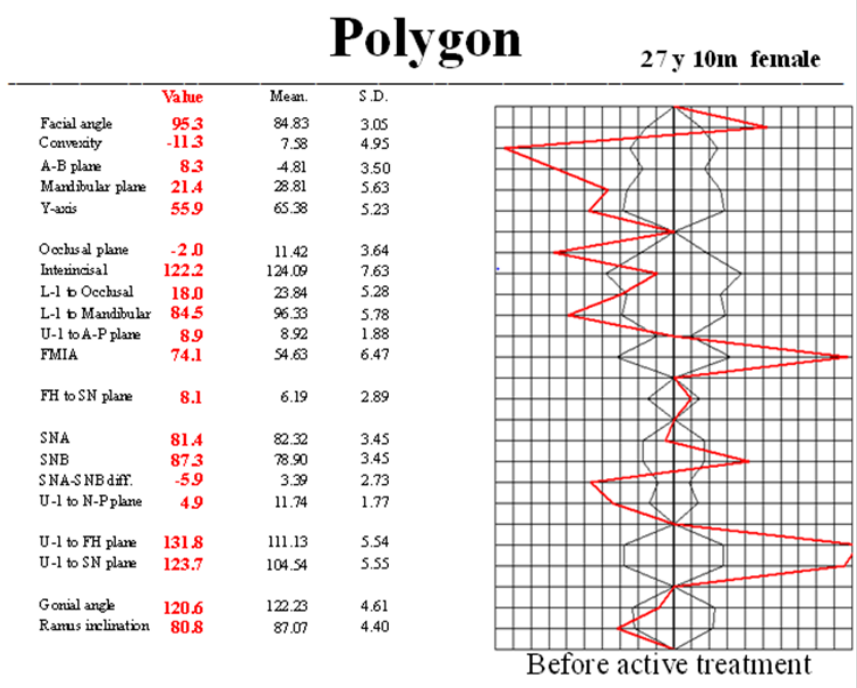

\section{Treatment Objectives}

Our treatment objectives were

i. To correct uneven occlusal plane and remove functional interference
ii. To improve facial profile
iii. To establish proper overbite, overjet and arch coordination
iv. To establish indivualized occlusion

\section{Treatment Progress}

Treatment was started from the maxillary arch. 0.018-inch readjusted edgewise appliances was bonded, and .016- X .022inch L\&H Titan Wire was used for initial leveling. L\&H Titan Wire expansion arch (Figure 7) was added over \#12 to \#15 area to resolve lateral cross bite and functional interference over right upper canine. The open coiled spring was used between \#25 and \#27 to create space for \#26 alignment. Shortly after lateral cross bite was corrected, mandibular arch was bonded. Intermaxillary elastics were used to adjust the jaw relationships and midline correction. After 10 months of active treatment, LH MEAW technique (Figure 8) was added to correct canted occlusion. After 13 months of active treatment, a functional appliance (FKO) was adopted as the retainer (Figure 9).

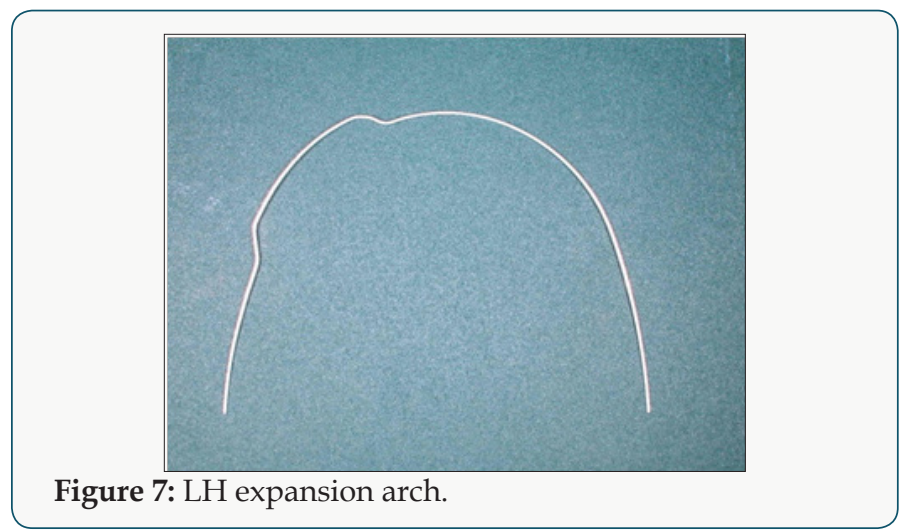

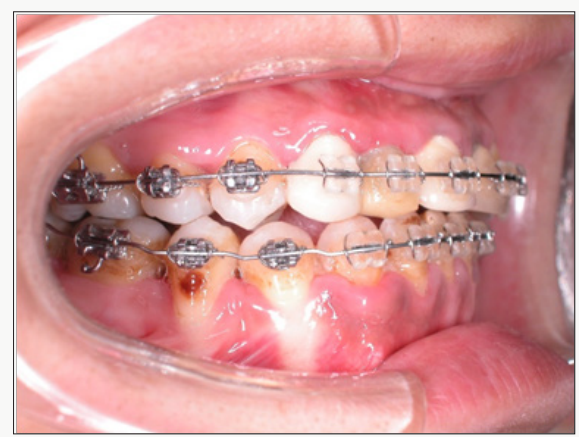

Figure 8: LH MEAW.

\section{Treatment Results}

After 13 months of active treatment, a stable occlusion was achieved, yet the asymmetrical appearance was not much improved immediately after the treatment (Figures 5 \& 6). After a retention period of 6 months by a functional appliance to maintain the intermaxillary relationship and to adjust the musculature tension, a new balanced combined with orthodontic treatment may be the better choice. But the patient refused the surgery. Before the orthodontic treatment was performed, differentially diagnosing a facial asymmetry case by dental/functional/skeletal is very crucial. Sometimes a case may combine two or more. For instance, this case showed both functional and skeletal problems. It was important to locate functional interference in a facial asymmetry case. Sometimes a functional wax bite was helpful. This case showed possibility of functional interference around the right upper canine. With LH (developed by the Tokyo Medical and Dental University) expansion arch around the canine area and trans-midline elastic traction, functional interference was relieved.

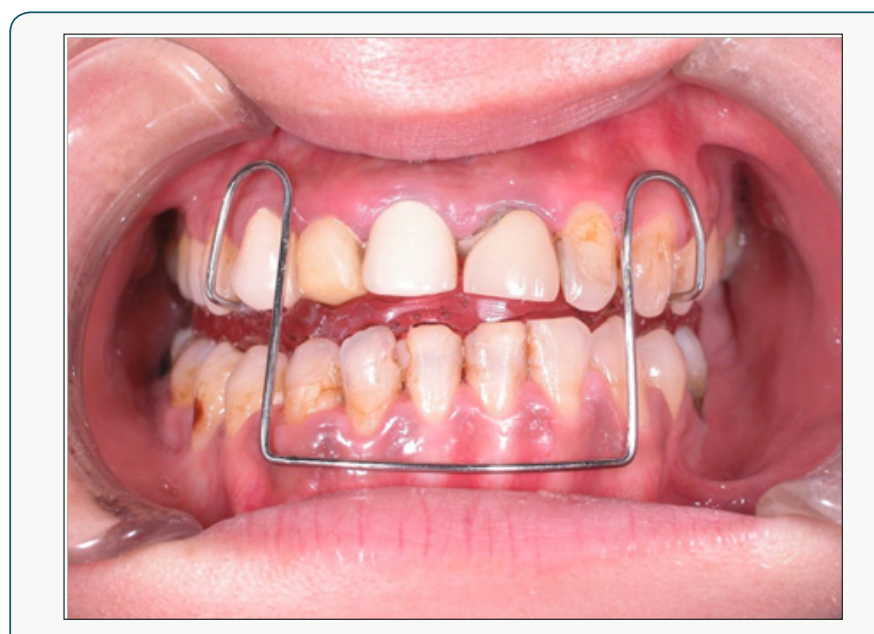

Figure 9: Functional appliance.

\section{Discussion}

The patient had skeletal class III malocclusion and facial asymmetry, orthognathic surgery LH MEAW was used to correct canted occlusion. LH wire with tip-back bends was added from canine to 2nd molar at upper left and lower right quadrants to correct canted occlusion. When we used LH wire with tip-back 
bends, we could expect upright and intrusion effect over posterior teeth and to correct uneven occlusion. Concerning retention strategy for a facial asymmetry case, in order to maintain a fixed intermaxillary relationship and to successfully relieve musculature tension, we adopted a functional appliance to keep a rigid and unchanged upper and lower jaw position. Although camouflage treatment may be available over some patients, we have to pay attention to their musculature tension to prevent the relapse.
CC (1) This work is licensed under Creative

To Submit Your Article Click Here: $\quad$ Submit Article

DOI: 10.32474/MADOHC.2018.01.000110

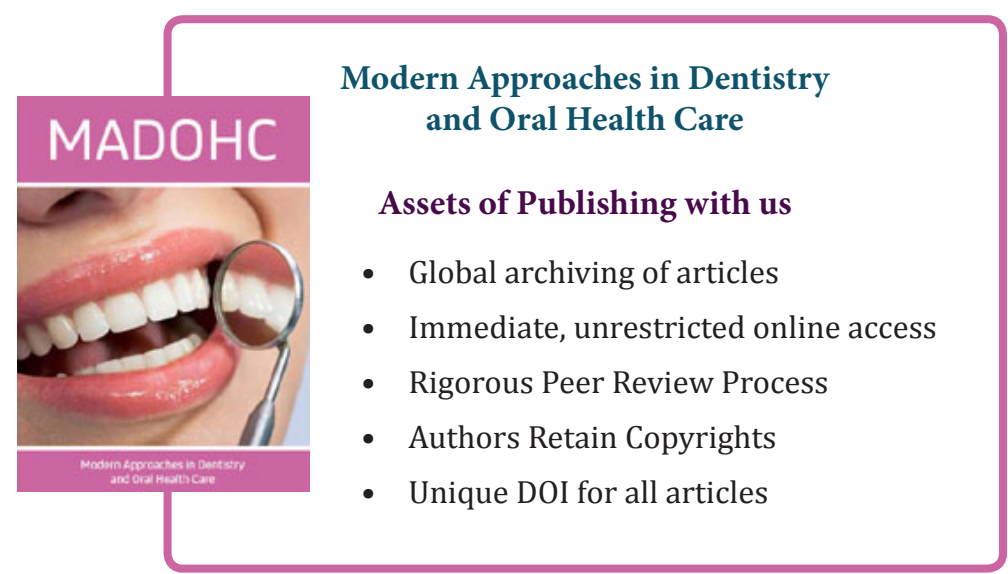

